\title{
Detection of Triphenylmethane Drugs in Fish Muscle by Surface-Enhanced Raman Spectroscopy Coupled with Au-Ag Core-Shell Nanoparticles
}

\author{
Lu Pei, ${ }^{1}$ Yiqun Huang, ${ }^{1,2}$ Chunying Li, ${ }^{1}$ Yuanyuan Zhang, \\ Barbara A. Rasco, ${ }^{2}$ and Keqiang Lai ${ }^{1}$ \\ ${ }^{1}$ College of Food Science and Technology, Shanghai Ocean University, No. 999 Hucheng Huan Road, \\ LinGang New City, Shanghai 201306, China \\ ${ }^{2}$ School of Food Science, Washington State University, Pullman, WA 99164, USA \\ Correspondence should be addressed to Keqiang Lai; kqlai@shou.edu.cn
}

Received 14 March 2014; Accepted 28 April 2014; Published 21 May 2014

Academic Editor: Yongfeng Luo

Copyright @ 2014 Lu Pei et al. This is an open access article distributed under the Creative Commons Attribution License, which permits unrestricted use, distribution, and reproduction in any medium, provided the original work is properly cited.

\begin{abstract}
Silver -coated gold bimetallic nanoparticles were synthesized and used as substrates for surface-enhanced Raman spectroscopy (SERS) in detecting prohibited triphenylmethane drugs (including crystal violet and malachite green) in fish muscle. The optical properties and physical properties of bimetallic nanospheres were characterized by UV-Vis spectroscopy and transmission electron microscopy. The optimal nanospheres selected had relatively uniform size (diameter: $33 \pm 3 \mathrm{~nm}$ ) with a silver layer coated on the surface of gold seed (diameter: $18 \pm 2 \mathrm{~nm}$ ). For both crystal violet and malachite green, characteristic SERS spectral features could be identified at concentration as low as $0.1 \mu \mathrm{g} / \mathrm{L}$ with these bimetallic nanospheres. Crystal violet and malachite green residues in fish muscle could also be detected at levels as low as $0.1 \mathrm{ng} / \mathrm{g}$, which could meet the most restricted regulatory requirements for the limit of detection in terms of analytical methods for crystal violet or malachite green in fish muscle. This study provides a basis for applying SERS technology with bimetallic nanoparticles to the identification of trace amounts of prohibited substances in aquatic food products, and the methodology could be extended to analyses of other hazardous chemicals in complex food matrices like vegetables and meats.
\end{abstract}

\section{Introduction}

Surface-enhanced Raman spectroscopy or surface-enhanced Raman scattering (SERS) utilizes the tremendous enhancement effect of Raman scattering signals through adsorbing analytes onto the roughened surfaces of gold, silver, and other metallic materials $[1,2]$. Chemical enhancement and electromagnetic enhancement are two accepted enhancement mechanisms, which contributed to the charge transfers between the adsorbed analyte molecule and metal substrate and the large local field enhancement in the vicinity of metal surfaces excited by the laser $[3,4]$. SERS has shown great potential for analyses of trace amounts of chemicals at as low as single molecule level [5-7]. In recent years, SERS technology has been increasingly exploited in applications in various fields, such as material, physics, medicine, and food science $[8,9]$. It is well known that SERS effect is quite sensitive to the changes in substrate materials and surface morphology. One of the key factors for successful applications of SERS technology is preparation of highly active, stable, and reproducible SERS substrates $[2,10]$. Au-Ag core-shell bimetallic nanoparticles (NPs) were synthesized by coating silver on the surface of gold seeds through chemical reduction. With appropriate particle sizes and thickness of silver shells, these bimetallic nanospheres could overcome problems, such as instability commonly tied to silver NPs and relatively low enhancement effects tied to gold NPs, and exhibit high enhancement effects similar to silver NPs with the advantages of high degree of homogeneity as gold NPs $[8,11-13]$. However, similar to other SERS substrates with great potential as analytical tools, the application aspect of bimetallic nanospheres as SERS substrates is left far behind 
the theory aspect of SERS related research and has become the bottleneck of the field.

Crystal violet (CV, 4-[bis(4-dimethylaminophenyl) methylidene]cyclohexa-2,5-dien-1-ylidene]-dimethylazanium chloride) and malachite green (MG, 4-[(4-dimethylaminophenyl)-phenyl-methyl]-N,N-dimethyl-aniline), both triphenylmethane dyes, are remarkably effective against fungal infections and parasitosis in fish and had been worldwide used as biocide and fungicide in aquaculture for decades [14]. Since mid-1980s, efforts have been made to reduce the use of both CV and MG due to their links to genotoxicity and carcinogenicity [15]. Although the use of these drugs has been banned in the United States, the European Union, China, and many other countries [16], CV and MG are still used illegally in some parts of the world due to their low cost and high efficiency, resulting in frequent occurrence of aquatic products with safety issues and consequently leading to import bans and product recalls. Based upon the Rapid Alert System for Food and Feed online searchable database of the European Union, a total of 131 cases of various fish products (such as catfish, trout, tilapia, salmon, king prawn, and caviar) contaminated with CV or MG were reported from 2003 to 2012 [17]. Food safety incidents like these have brought huge barriers to import and export trades of global aquatic products, so it is particularly important to strengthen the monitoring system for $\mathrm{CV}$ and $\mathrm{MG}$ residues in aquatic products. High performance liquid chromatography (HPLC) is the most commonly used method for analyzing $\mathrm{CV}$ and MG drug residues in fish, but it has disadvantages such as high cost, time consuming, and too complicated sample preparation [18]. In addition, with the increasing tightened policy towards CV and MG, the sensitivity of HPLC method is unlikely to achieve the limit of detection at $1 \mathrm{ng} / \mathrm{g}$ level required by most of the countries that ban the drugs, and consequently not only more sensitive but also much costly liquid chromatography-mass spectrometry (LC-MS) is required for detecting CV or MG at such low level [19]. SERS technology has shown great potential for detecting trace amounts of analytes with a simpler sample extraction protocol and a shorter detection time, which makes it possible to rapidly screen and analyze the use of illegal drugs in aquatic products [20].

The objective of this study was to investigate the potential of applying Au-Ag core-shell bimetallic nanoparticles to the detection of banned triphenylmethane drugs in fish muscle. This study provides a basis for applying SERS technology to the identification of trace amounts of prohibited substances in aquatic food products, and the methodology could be extended to analyses of other hazardous chemicals in complex food matrices (such as fish and meat) with SERS technology.

\section{Materials and Methods}

2.1. Synthesis of Au-Ag Core-Shell Nanoparticles. Au-Ag coreshell NPs were synthesized in solution via a seed-growth method [21]. In brief, sodium citrate $(0.74 \mathrm{~mL}, 1 \% \mathrm{w} / \mathrm{w})$ was added to the boiling solution of chloroauric acid $(50 \mathrm{~mL}$, $2 \times 10^{-4} \mathrm{~mol} / \mathrm{L}$ ), and the mixture was stirred and boiled until the color became wine red, indicating that Au NPs were formed [22]. As-prepared Au colloids $(3 \mathrm{~mL})$ and Lascorbic acid $(0.4 \mathrm{~mL}, 0.1 \mathrm{~mol} / \mathrm{L})$ were added in a vial under continuous stirring. Then $0.3 \mathrm{~mL}, 0.6 \mathrm{~mL}, 0.9 \mathrm{~mL}$, and $1.2 \mathrm{~mL}$ of silver nitrate $\left(1 \times 10^{-3} \mathrm{~mol} / \mathrm{L}\right)$ were added dropwise $(10 \mu \mathrm{L}$ per addition) to this mixture to prepare four different $\mathrm{Au}$ Ag core-shell NPs varying in the thickness of silver coating, respectively. The formed $\mathrm{Au}-\mathrm{Ag}$ core-shell NPs were transferred into a conical flask with stopper and kept in refrigerator at $4^{\circ} \mathrm{C}$ before use.

2.2. Preparation of Standard Solutions. CV ( $\geq 90 \%$, SigmaAldrich, USA) and MG ( $>99 \%$, Sigma-Aldrich, USA) were dissolved in acetonitrile (HPLC reagent, Sigma, USA) aqueous solution $(\mathrm{v} / \mathrm{v}=1: 1)$ to prepare a series of standard solutions $\left(0.1,1,10\right.$, and $\left.10^{3} \mu \mathrm{g} / \mathrm{L}\right)$. The $\mathrm{pH}$ of the $\mathrm{CV}$ and MG standard solutions ranged from 5.3 to 5.8, depending on the concentration of the drugs. The absorbance bands of $\mathrm{CV}$ and MG were at $590 \mathrm{~nm}$ and $620 \mathrm{~nm}$, respectively, based upon UV-Vis spectroscopic analysis (UV3000PC, MAPADA Instruments Ltd., Shanghai, China).

2.3. Fish Sample Pretreatment. Tilapia fillets (containing no $\mathrm{CV}$ or MG as confirmed with LC-MS) from Zhenye Aquatic and Cool Storage Ltd. (Guangdong, China) were used in this study. Tilapia fillets were homogenized through blending frozen tilapia filets with dry ice in a laboratory blender (HGBTWTS3, Waring Commercial, Torrington, CT, USA) at high speed for 5 minutes to achieve uniform fish samples and therefore minimize spectral variation due to the disparity of nontargeted components in fish tissue. The extraction and purification protocol for $\mathrm{CV}$ and MG in fish muscle were based upon a conventional method adopted by the US Food and Drug Administration (FDA) with slight modifications [23]. In brief, homogenized tilapia fillets spiked with CV or MG [0 (blank), 0.1, 1 , and $10 \mathrm{ng} / \mathrm{g}$ ] were blended with ammonium acetate buffer (Sinopharm Chemical Reagent Ltd., SCRC, Shanghai, China), hydroxylamine hydrochloride solution (ACS reagent, SCRC), and p-toluenesulfonic acid solution (ACS reagent, SCRC) and mixed well. Then, acetonitrile and alumina (chromatographic grade, SCRC) were added and the mixture was centrifuged, followed by the use of supernatants for liquid-liquid extraction with the addition of dichloromethane (ACS reagent, SCRC). The extract was evaporated to dry with a rotary evaporator (R206B, Shanghai SENCO Technology Ltd., Shanghai, China), dissolved into acetonitrile, and mixed with 2,3-dichloro-5,6-dicyano-1,4benzoquinone (DDQ) solution (98\%; J\&K SCIENTIFIC, Logan, UT, USA). The solution was further purified with solid phase extraction through alumina cartridge $(1 \mathrm{~g}, 3 \mathrm{~mL}$; Supelco, Bellefonte, PA, USA) positioned on top of a propylsulfonic acid cartridge $(500 \mathrm{mg}, 3 \mathrm{~mL}$; ANPEL Scientific Instrument Co., Ltd., Shanghai, China). Unlike the FDA method, no vacuum pump was used to speed up the flow rate during the solid phase extraction. The final elute was reconstituted to $5 \mathrm{~mL}$ with ammonium acetate and acetonitrile (v/v $=1: 1$ ).

2.4. SERS Measurement. SERS spectra were collected by using a Nicolet DXR microscopy Raman spectrometer 
(Thermo Fisher Scientific Inc., Waltham, MA, USA) with a $633 \mathrm{~nm}$ He-Ne laser source, $2 \mathrm{~mW}$ laser power, and 20x objective lens with a slit width of $50 \mathrm{~cm}^{-1}$.

To collect an SERS spectrum, $10 \mu \mathrm{L}$ Au-Ag core-shell NPs were deposited onto a microscope glass slide, and after evaporation of solvent, one drop of sample solution or fish extract was pipetted onto the $\mathrm{Au}-\mathrm{Ag}$ core-shell substrate. SERS spectra were immediately acquired after evaporation of the solvent. The exposure time was $20 \mathrm{~s}$ for each scan and each spectrum was the average of 5 scans. For each treatment of standard solutions $\left(10^{3}, 10,1\right.$, and $\left.0.1 \mu \mathrm{g} / \mathrm{L}\right)$ or fish fillets spiked with CV or MG [0 (blank), 0.1, 1, and $10 \mathrm{ng} / \mathrm{g}], 10$ spectra from different locations of a substrate were collected, and triplicate analyses were conducted.

2.5. Characterizations of Nanoparticles. The optical properties of Au NPs used as seeds and Au-Ag core-shell NPs coated with different amounts of silver were analyzed with a UV-Vis absorbance spectroscopy (UV3000PC, MAPADA Instruments Ltd., Shanghai, China). General shapes of the Au and $\mathrm{Au}-\mathrm{Ag}$ core-shell NPs were analyzed with a transmission electron microscopy (TEM; JEM-2100F, JEOL Ltd., Tokyo, Japan) and the core-shell structure of Au-Ag core-shell NPs was determined with a high-resolution TEM (HRTEM, JEM2100F, JEOL Ltd., Tokyo, Japan). The average particle sizes of Au seeds and bimetallic NPs were calculated based upon TEM images of 50 particles.

CV standard solution $(10 \mu \mathrm{g} / \mathrm{L})$ was used as probe to understand the effect of the synthesized Au-Ag core-shell substrates on SERS enhancement. The SERS spectra of CV with the use of four different Au-Ag core-shell NPs as substrates were recorded, respectively, and the enhancement factors of these substrates were estimated and compared [24]. The substrate resulting in the highest enhancement for CV was selected for the further study on analysis of CV and MG standard solutions and fish fillets spiked with CV or MG.

To evaluate the stability of Au-Ag core-shell bimetallic nanosphere colloids, the selected $\mathrm{Au}-\mathrm{Ag}$ core-shell NPs were stored at refrigerator temperature for up to 20 days. Sixteen spectra of CV standard solution $(1 \mu \mathrm{g} / \mathrm{L}, 10 \mu \mathrm{g} / \mathrm{L})$ with the use of the selected colloids as SERS substrates were acquired every other day. The Raman intensities at $1617 \mathrm{~cm}^{-1}$ (one of the primary characteristic peaks for $\mathrm{CV}$ ) were compared among spectra collected from different days to see whether there was significant difference $(\alpha=0.05)$ with ANOVA analysis (Excel 2010; Microsoft Co., Redmond, WA, USA).

\section{Results and Discussion}

3.1. UV-Vis Spectra of Au-Ag Core-Shell NPs with Different Sizes. The surface plasmon resonance (SPR) peak of metal NPs can be detected with UV-Vis absorbance spectroscopy. It is well known that the position and shape of SPR peak depend upon the shape, size, and composition of NPs [25], and all these factors ultimately affect the enhancement effect of SERS substrates. The reported SPR peaks of Au NPs ranged from $517 \mathrm{~nm}$ to $575 \mathrm{~nm}$ (particle diameter: 9-99 nm), while the peaks of Ag NPs ranged from $390 \mathrm{~nm}$ to $438 \mathrm{~nm}$ (particle diameter: $10-80 \mathrm{~nm}$ ), and the SPR peak tended to shift to

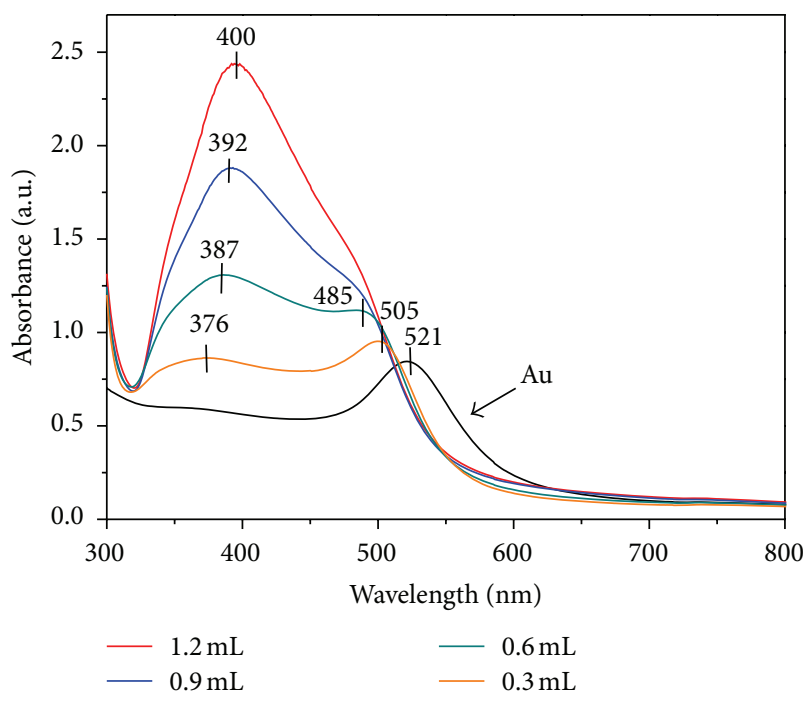

FIGURE 1: Changes of UV-Vis spectra of Au-Ag core-shell nanoparticles dispersion with the increased amounts of $\mathrm{AgNO}_{3}$ used for synthesis.

higher wavelength (red shift) with an increase in particle sizes for Au or Ag NPs [26-28]. The optical properties of Au-Ag core-shell NPs are more complex than those of $\mathrm{Au}$ or $\mathrm{Ag}$ NPs because of an interaction between $\mathrm{Au}$ and $\mathrm{Ag}$. As shown in Figure 1, the change in the plasmon resonance of $\mathrm{Au}-\mathrm{Ag}$ core-shell NPs during the silver coating process was clearly affected by the ratio of $\mathrm{Au}$ to $\mathrm{Ag}$ and the thickness of $\mathrm{Ag}$ shell. Before adding $\mathrm{AgNO}_{3}$, Au NPs dispersion exhibited absorbance band at about $521 \mathrm{~nm}$, and the shape of the SPR peak was symmetrical and narrow, indicating relatively good monodispersion of the Au NPs (diameter: $18 \pm 2 \mathrm{~nm}$ ). With the addition of $\mathrm{AgNO}_{3}$ solution and ascorbic acid, $\mathrm{Ag}^{0}$ was produced and gradually coated on the surface of Au seeds as indicated by the appearance of characteristic peak of silver shells (376-400 nm) as well as the shifts of SPR peaks for both $\mathrm{Ag}$ shells and $\mathrm{Au}$ seeds (Figure 1). As the amount of $\mathrm{AgNO}_{3}$ solution increased from $0.3 \mathrm{~mL}$ to $1.2 \mathrm{~mL}$, the SPR peak of Ag shells red-shifted from $376 \mathrm{~nm}$ to $400 \mathrm{~nm}$ and the peak intensity also strengthened due to increase in the thickness of silver layer deposited onto the Au seeds [29], while the SPR peak for Au seeds showed opposite trend and was barely discernible when the amount of $\mathrm{AgNO}_{3}$ solution reached up to $0.9 \mathrm{~mL}$ (particle diameter: $33 \pm 3 \mathrm{~nm}$ ) and above. The overall SPR peaks of Ag shells for Au-Ag core-shell NPs appeared at relatively low wavelengths $(376-400 \mathrm{~nm})$ compared to those reported for Ag NPs (390-438 nm), which was mainly because the Ag shells (5-11 nm) were quite thin compared to the radiuses of the reported Ag NPs $(5-40 \mathrm{~nm})[27,28]$.

3.2. Nanoparticles Size-Dependent SERS Enhancement Effect for $C V$. Figure 2 shows some representative SERS spectra of $10 \mu \mathrm{g} / \mathrm{L} \mathrm{CV}$ standard solution acquired by using four $\mathrm{Au}-\mathrm{Ag}$ core-shell substrates synthesized with different amounts of $\mathrm{AgNO}_{3}$. The observed main characteristic peaks of $\mathrm{CV}$ at around $1617,1367,1173,914$, and $440 \mathrm{~cm}^{-1}$ are assigned to the 


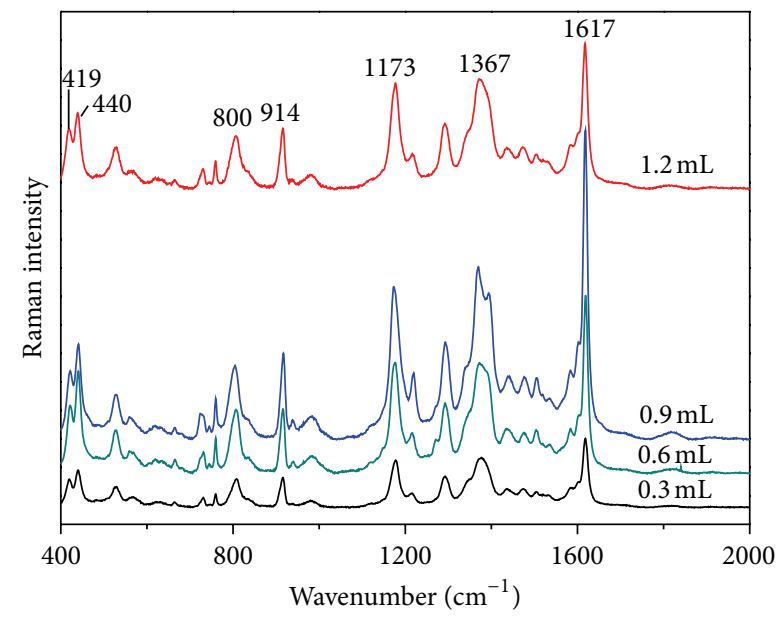

FIGURE 2: Representative SERS spectra of $10 \mu \mathrm{g} / \mathrm{L}$ crystal violet solution by using Au-Ag core-shell substrates synthesized with different amounts of $\mathrm{AgNO}_{3}$.

in-plane stretching of the ring- $\mathrm{C}-\mathrm{C}, \mathrm{N}-$ phenyl stretching, in-plane bending of the ring-C-H, ring skeletal vibration of radical orientation, and out-of-plane deformation vibrations of the phenyl- $\mathrm{C}^{+}$-phenyl $[30,31]$. As can be seen from the change in the intensity of CV characteristic peaks, an increasing enhancement effect was observed with the use of thicker silver-coated Au-Ag core-shell NPs when the amount of $\mathrm{AgNO}_{3}$ used for silver coating was no more than $0.9 \mathrm{~mL}$. The Au-Ag core-shell NPs could reach sufficiently intrinsic SERS activity of the growing Ag shells, which is much higher than $\mathrm{Au}$ NPs to generate strong electromagnetic enhancement for high SERS signals [32]. However, the enhancement effect decreased when the amount of $\mathrm{AgNO}_{3}$ used for silver coating reached $1.2 \mathrm{~mL}$. The enhancement factors for four $\mathrm{Au}-$ Ag core-shell substrates were calculated as $2.5 \times 10^{5}, 8.0 \times$ $10^{5}, 1.8 \times 10^{6}$, and $6.1 \times 10^{5}$, corresponding to $0.3,0.6,0.9$, and $1.2 \mathrm{~mL}$ of $\mathrm{AgNO}_{3}$ used for silver coating, respectively. Therefore, the Au-Ag core-shell NPs synthesized with $0.9 \mathrm{~mL}$ of $\mathrm{AgNO}_{3}$ (particle diameter: $33 \pm 3 \mathrm{~nm}$ ) were selected for further analysis of $\mathrm{CV}$ and $\mathrm{MG}$ in fish muscle.

Based on the calculation of the TEM images of 50 particles, the average particle size of Au seeds was $18 \pm 2 \mathrm{~nm}$ in diameter, and the sizes of Au-Ag core-shell NPs were $28 \pm$ $4 \mathrm{~nm}, 30 \pm 4 \mathrm{~nm}, 33 \pm 3 \mathrm{~nm}$, and $39 \pm 3 \mathrm{~nm}$, corresponding to $0.3,0.6,0.9$, and $1.2 \mathrm{~mL}$ of $\mathrm{AgNO}_{3}$ used for silver coating, respectively. The TEM images of the Au seeds and the $33 \mathrm{~nm}$ $\mathrm{Au}-\mathrm{Ag}$ core-shell NPs showed that both NPs were relatively uniform, nearly spherical with narrow size distribution (relative standard deviation: Au NPs, $11 \% ; 33 \pm 3 \mathrm{~nm} \mathrm{Au-Ag}$ coreshell NPs, 9\%) (Figures 3(a) and 3(b)). The average thickness of the Ag layer on the Au core is $7.5 \mathrm{~nm}$ for the selected $\mathrm{Au}-\mathrm{Ag}$ core-shell NPs. In addition, no seed-size Au NPs or smaller Ag NPs were observed, suggesting that no other nucleation centers or separation from the Au-core layer occurred $[6,33]$. The HRTEM image of Au-Ag core-shell NPs (Figure 3(c)) showed that some lateral moire fringes were generated which indicated the selective growing of $\mathrm{Ag}$ [34-36].
During 20-day storage at refrigerator temperature, with the selected $33 \mathrm{~nm} \mathrm{Au-Ag}$ core-shell NPs as substrates to acquire SERS spectra of CV standard solution $(1 \mu \mathrm{g} / \mathrm{L}$, $10 \mu \mathrm{g} / \mathrm{L}$ ), there was no significant change in the Raman intensities at $1617 \mathrm{~cm}^{-1}$ for both $1 \mu \mathrm{g} / \mathrm{L}$ and $10 \mu \mathrm{g} / \mathrm{L}$ solutions, indicating that the colloids were stable and no obvious change occurred in 20 days.

\subsection{Analysis of $C V$ and $M G$ with Au-Ag Core-Shell SERS} Substrates. A series of CV and MG standard solution (0.1, 1,10 , and $10^{3} \mu \mathrm{g} / \mathrm{L}$ ) was used to evaluate the SERS activity of the selected $33 \mathrm{~nm}$ Au-Ag core-shell substrates. As can be seen from Figure 4, there was almost no SERS signal for blank substrates, whereas the intensity of the characteristic bands was drastically enhanced as the concentration of CV or MG solution increased from $0.1 \mu \mathrm{g} / \mathrm{L}$ to $10 \mu \mathrm{g} / \mathrm{L}$. Even at the concentration level as low as $0.1 \mu \mathrm{g} / \mathrm{L}$, the major characteristic bands at around 440,914, 1173, $1367 \mathrm{~cm}^{-1}$, and $1617 \mathrm{~cm}^{-1}$ could still be identified for both CV and MG. SERS spectra of MG were similar to those of CV because of their similar molecular structures, and only changes in the relative intensity among peaks were observed, such that the relative intensity of the peak at around $1217 \mathrm{~cm}^{-1}$ for MG was stronger than that for CV $[20,37]$.

The optimal Au-Ag core-shell substrates were further used to detect $\mathrm{CV}$ and $\mathrm{MG}$ residues in fish samples with conventional sample preparation method. Figure 5 presents SERS spectra of CV and MG extracts [0 (blank), 0.1, 1, and $10 \mathrm{ng} / \mathrm{g}$ ] adsorbed on Au-Ag core-shell substrates. There was no SERS signal detected for the blank extracts, which indicates that sample extract was relatively clean and the presence of some nontargeted compounds did not have obvious Raman scattering signals that may interfere with the analysis of CV or MG. The main spectral characteristic peaks of fish extracts with different levels of CV or MG were basically consistent with those of the standard solution, showing similar intensity and no obvious interference by other components from fish fillets. In addition, the major characteristic bands were discernable at a level as low as $0.1 \mathrm{ng} / \mathrm{g}$ for both CV and MG in contaminated fish samples, which were similar to the results for CV or MG standard solutions as discussed earlier. A high recovery rate (85.9-93.9\%) was obtained by using the conventional methods for CV and MG extraction [38]. This high recovery rate of $\mathrm{CV}$ and $\mathrm{MG}$ together with little or no interference of other components in CV and MG extracts accounted for the similar SERS results for analysis of CV or MG in fish extracts and in standard solutions.

Obtaining reproducible spectra has been always a challenge for applying SERS technology as an analytical tool, since the intensities of SERS signals were greatly affected by the surface morphology of substrates, location, and direction of the targeted molecules adsorbed on a substrate and many other factors. To achieve reproducible results, ten spectra were acquired from different locations of a substrate each time during SERS collection, and the average of these ten spectra was used as the final spectra. Figure 6 exhibits the average spectra from triplicate analyses for CV and MG of both standard solutions $(1 \mu \mathrm{g} / \mathrm{L})$ and fish extracts $(1 \mathrm{ng} / \mathrm{g})$, 


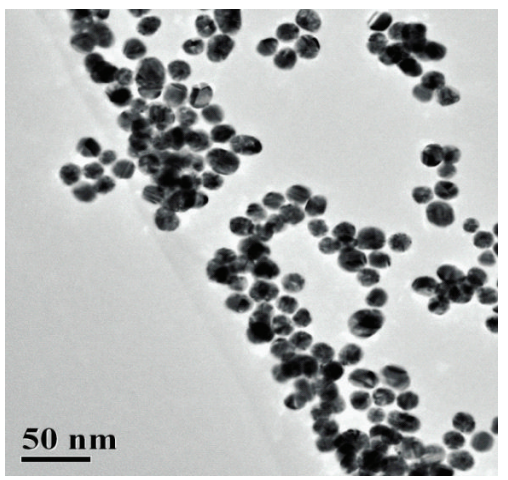

(a)

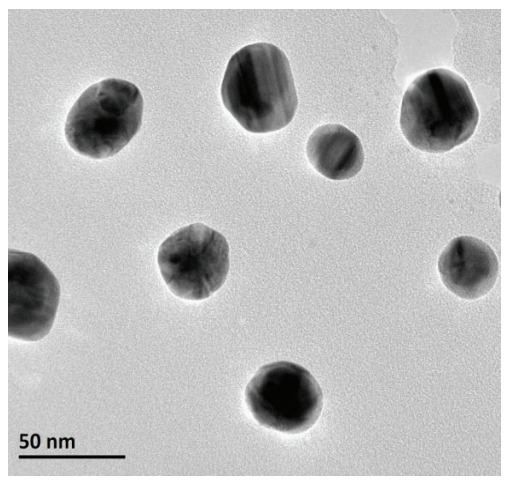

(b)

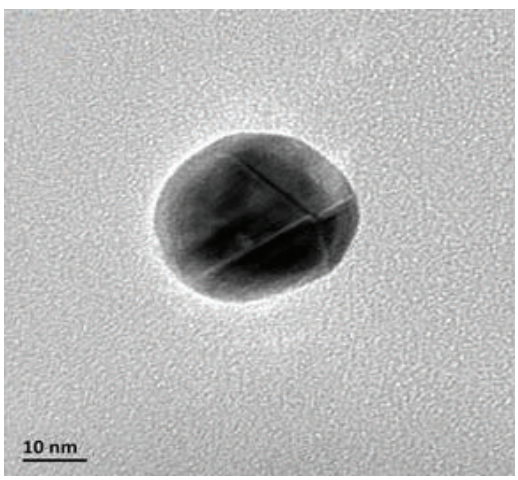

(c)

Figure 3: TEM images of (a) Au nanoparticles and (b) Au-Ag core-shell nanoparticles (0.9 mL AgNO 3 used) and (c) HRTEM image of Au-Ag core-shell nanoparticles (0.9 $\mathrm{mL} \mathrm{AgNO}_{3}$ used).

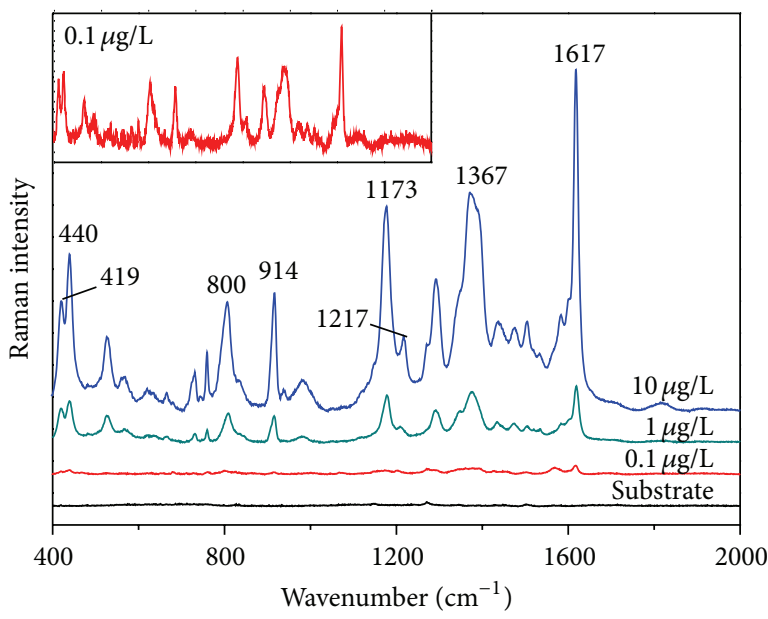

(a)

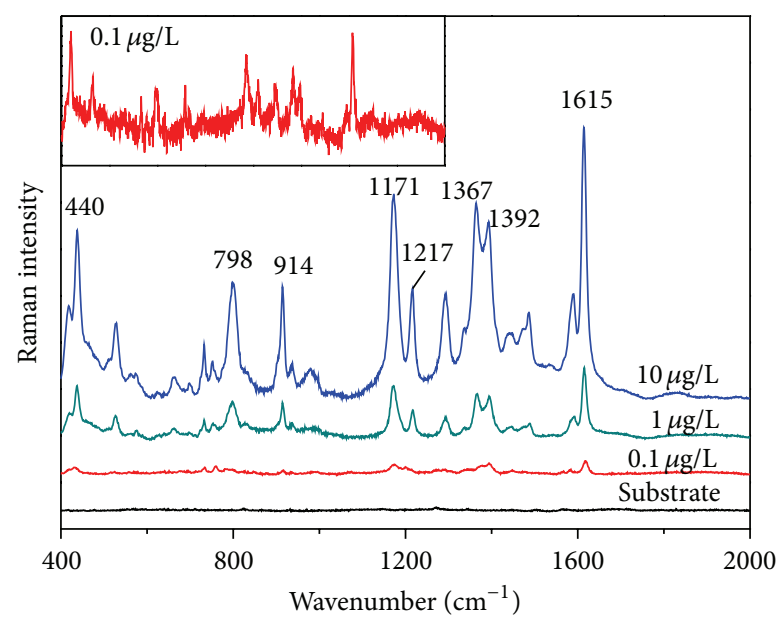

(b)

FIGURE 4: SERS spectra of (a) crystal violet standard solution and (b) malachite green standard solution deposited on Au-Ag core-shell nanoparticles.

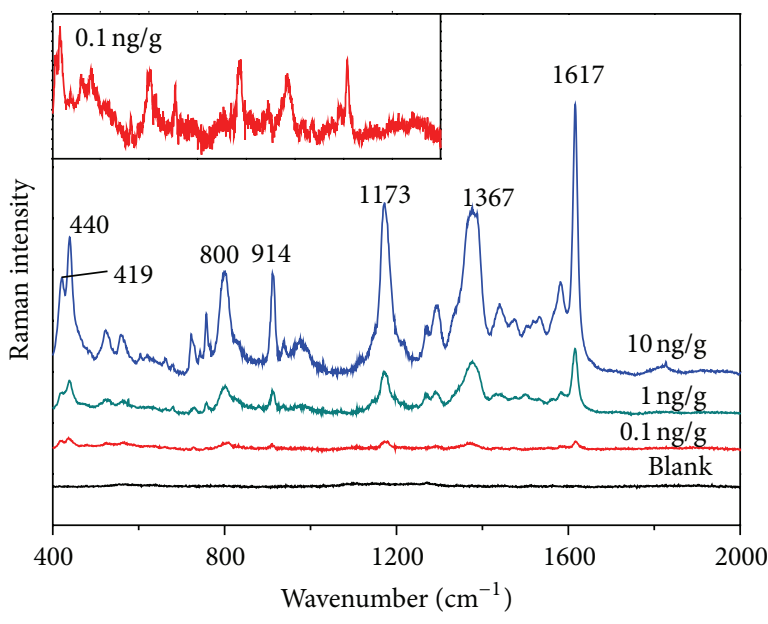

(a)

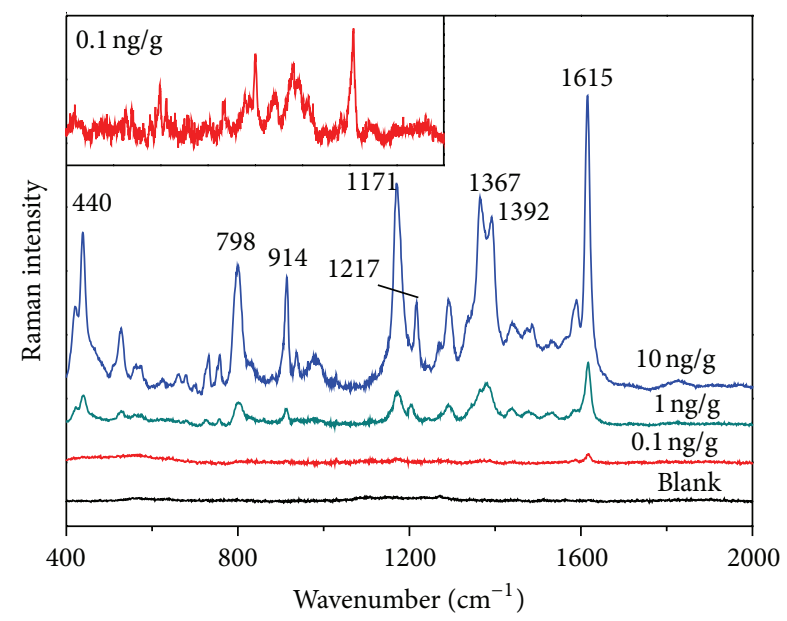

(b)

FIGURE 5: SERS spectra of (a) crystal violet extracts and (b) malachite green extracts from fish muscle. 


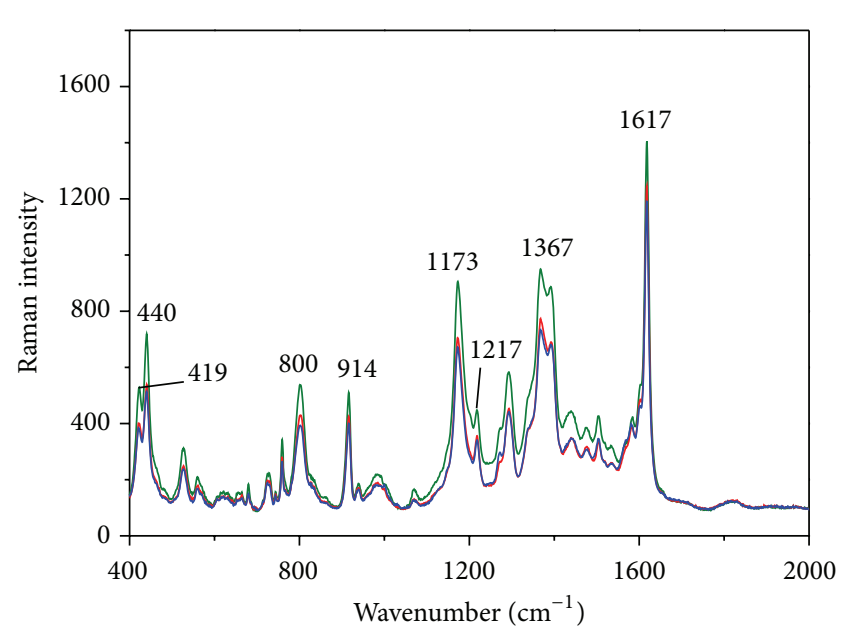

(a)

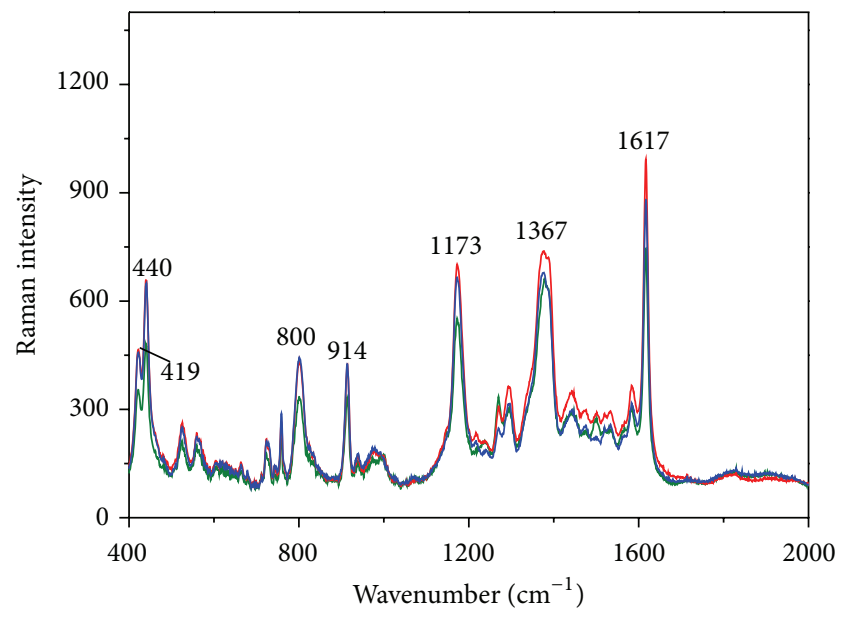

(c)

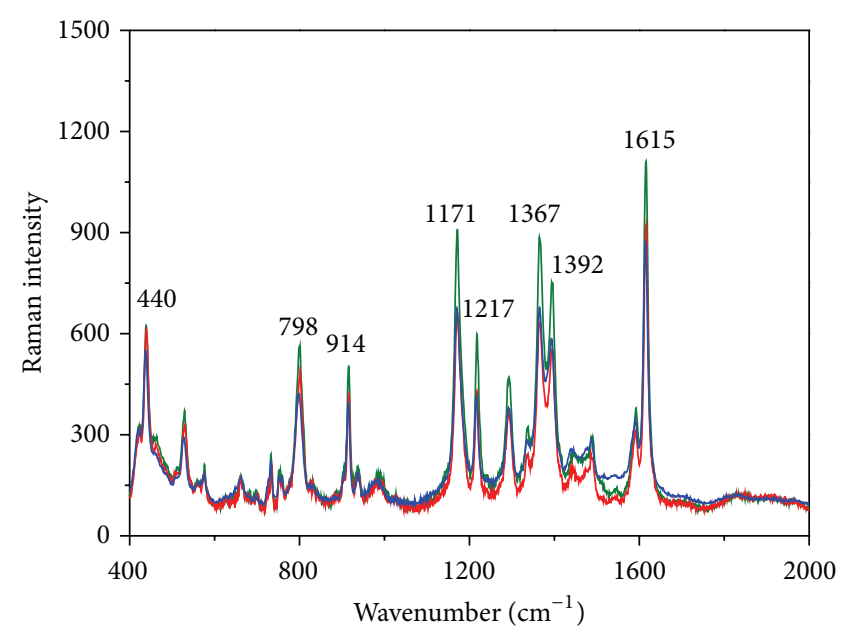

(b)

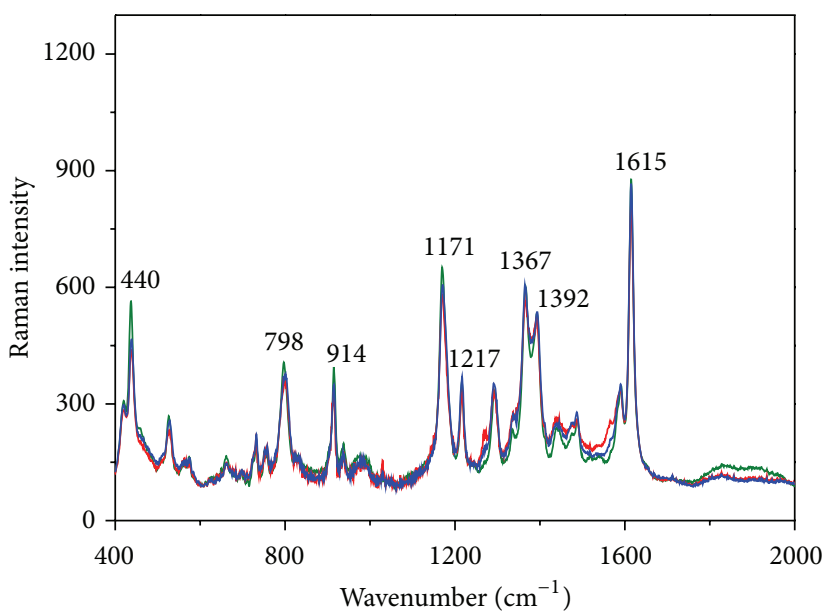

(d)

FIGURE 6: Average SERS spectra ( $n=10)$ of (a) CV standard solutions ( $1 \mu \mathrm{g} / \mathrm{L}),(\mathrm{b}) \mathrm{MG}$ standard solutions $(1 \mu \mathrm{g} / \mathrm{L})$, (c) CV fish extract (1 ng/g), and (d) MG fish extract (1 ng/g) from triplicate analyses.

indicating that relatively repeatable SERS spectra could be achieved with the selected Au-Ag core-shell NPs as substrates.

Most of CV or MG dyes are metabolized to leucocrystal violet or leucomalachite green in fish [19]. The conventional sample preparation method applied in this study to extract CV or MG from fish fillet involved the use of DDQ to convert metabolites leucocrystal violet or leucomalachite green to $\mathrm{CV}$ or $\mathrm{MG}$, and the final level of fish drug detected was actually the sum of CV (or MG) and its metabolite. Therefore, although only CV or MG was tested, the study results could be applied to analyze CV (or MG) and its metabolite in fish muscle.

\section{Conclusions}

Silver-coated gold bimetallic NPs were synthesized and successfully applied to detect as low as $0.1 \mathrm{ng} / \mathrm{g}$ of CV or MG in fish muscle, which made great improvement over our previous studies using commercial SERS substrates [20] and also could meet the most restricted regulatory requirements for the $1 \mathrm{ng} / \mathrm{g}$ limit of detection for crystal violet or malachite green in fish muscle. This interdisciplinary study combined knowledge in SERS and analytical chemistry to solve a real world food safety problem, which helps to fill the gap between theory and application aspects of SERS-related research. Although there were reports on using SERS for nondestructive analyses of chemical hazards in food, such as pesticide in apples [35], since CV or MG residues are inside fish tissues, it is impossible to apply SERS technology to analyze these drugs without sample preparation involving extraction and purification. In this study, a general sample preparation method for chromatography-based analyses was adopted; the information gained from the study provides a basis for further explorations on simplifying sample preparation methods as well as for expanding SERS methods with bimetallic nanospheres for analysis of other harmful chemical substances, such as pesticides and antibiotics in food systems.

\section{Conflict of Interests}

The authors declare that there is no conflict of interests regarding the publication of this paper. 


\section{Acknowledgment}

This research was supported by the National Natural Science Foundation of China (61250002 and 31250006).

\section{References}

[1] D. L. Jeanmaire and R. P. van Duyne, "Surface Raman spectroelectrochemistry-part I: heterocyclic, aromatic, and aliphatic amines adsorbed on the anodized silver electrode," Journal of Electroanalytical Chemistry and Interfacial Electrochemistry, vol. 84, no. 1, pp. 1-20, 1977.

[2] C. L. Haynes, A. D. McFarland, and R. P. van Duyne, "Surfaceenhanced Raman spectroscopy," Analytical Chemistry A, vol. 77, no. 17, pp. 338-346, 2005.

[3] E. Petryayeva and U. J. Krull, "Localized surface plasmon resonance: nanostructures, bioassays and biosensing-a review," Analytica Chimica Acta, vol. 706, no. 1, pp. 8-24, 2011.

[4] D. Cialla, A. März, R. Böhme et al., "Surface-enhanced Raman spectroscopy (SERS): progress and trends," Analytical and Bioanalytical Chemistry, vol. 403, no. 1, pp. 27-54, 2012.

[5] S. Nie, "Probing single molecules and single nanoparticles by surface-enhanced Raman scattering," Science, vol. 275, no. 5303, pp. 1102-1106, 1997.

[6] M. Mandal, N. R. Jana, S. Kundu, S. K. Ghosh, M. Panigrahi, and T. Pal, "Synthesis of Aucore-Agshell type bimetallic nanoparticles for single molecule detection in solution by SERS method," Journal of Nanoparticle Research, vol. 6, no. 1, pp. 53-61, 2004.

[7] M. V. Cañamares, C. Chenal, R. L. Birke, and J. R. Lombardi, "DFT, SERS, and single-molecule SERS of crystal violet," The Journal of Physical Chemistry C, vol. 112, no. 51, pp. 2029520300, 2008.

[8] L. Lu, H. Wang, Y. Zhou et al., "Seed-mediated growth of large, monodisperse core-shell gold-silver nanoparticles with Ag-like optical properties," Chemical Communications, no. 2, pp. 144145, 2002.

[9] F. Zhai, Y. Huang, C. Li, X. Wang, and K. Lai, "Rapid determination of ractopamine in swine urine using surface-enhanced raman spectroscopy," Journal of Agricultural and Food Chemistry, vol. 59, no. 18, pp. 10023-10027, 2011.

[10] Y. Li, H. Su, K. S. Wong, and X.-Y. Li, "Surface-enhanced raman spectroscopy on two-dimensional networks of gold nanoparticle-nanocavity dual structures supported on dielectric nanosieves," The Journal of Physical Chemistry C, vol. 114, no. 23, pp. 10463-10477, 2010.

[11] N. R. Jana, "Silver coated gold nanoparticles as new surface enhanced Raman substrate at low analyte concentration," Analyst, vol. 128, no. 7, pp. 954-956, 2003.

[12] Y. Cui, B. Ren, J.-L. Yao, R.-A. Gu, and Z.-Q. Tian, "Synthesis of AgcoreAushell bimetallic nanoparticles for immunoassay based on surface-enhanced Raman spectroscopy," The Journal of Physical Chemistry B, vol. 110, no. 9, pp. 4002-4006, 2006.

[13] L. Guerrini, J. V. Garcia-Ramos, C. Domingo, and S. SanchezCortes, "Ultrathin silver-coated gold nanoparticles as suitable substrate for surface-enhanced raman scattering," Journal of Raman Spectroscopy, vol. 41, no. 5, pp. 508-515, 2010.

[14] S. Srivastava, R. Sinha, and D. Roy, "Toxicological effects of malachite green," Aquatic Toxicology, vol. 66, no. 3, pp. 319-329, 2004.

[15] N. A. Littlefield, B. N. Blackwell, C. C. Hewitt, and D. W. Gaylor, "A novel surface-enhanced Raman scattering sensor to detect prohibited colorants in food by graphene/silver nanocomposite," Fundamental and Applied Toxicology, vol. 5, pp. 902-912, 1985.

[16] A. Stammati, C. Nebbia, I. de Angelis et al., "Effects of malachite green $(\mathrm{MG})$ and its major metabolite, leucomalachite green (LMG), in two human cell lines," Toxicology in Vitro, vol. 19, no. 7, pp. 853-858, 2005.

[17] RAFFS, Subject: crystal violet or malachite green. Notification between 01/01/2003 to 31/12/2012, 2013, https://webgate.ec https://webgate.ec.europa.eu/rasff-window/portal.

[18] A. A. Bergwerff and P. Scherpenisse, "Determination of residues of malachite green in aquatic animals," Journal of Chromatography B: Analytical Technologies in the Biomedical and Life Sciences, vol. 788, no. 2, pp. 351-359, 2003.

[19] W. C. Andersen, S. B. Turnipseed, C. M. Karbiwnyk et al., "Multiresidue method for the triphenylmethane dyes in fish: malachite green, crystal (gentian) violet, and brilliant green," Analytica Chimica Acta, vol. 637, no. 1-2, pp. 279-289, 2009.

[20] Y. Zhang, K. Lai, J. Zhou, X. Wang, B. A. Rasco, and Y. Huang, "A novel approach to determine leucomalachite green and malachite green in fish fillets with surface-enhanced Raman spectroscopy (SERS) and multivariate analyses," Journal of Raman Spectroscopy, pp. 1208-1213, 2012.

[21] T. Y. Olson, A. M. Schwartzberg, C. A. Orme, C. E. Talley, B. O'Conneull, and J. Z. Zhang, "Hollow gold-silver doubleshell nanospheres: structure, optical absorption, and surfaceenhanced Raman scattering," The Journal of Physical Chemistry C, vol. 112, no. 16, pp. 6319-6329, 2008.

[22] G. Frens, "Controlled nucleation for the regulation of the particle size in monodisperse gold suspensions," Nature Physical Science, vol. 241, pp. 20-22, 1973.

[23] W. C. Andersen, S. B. Turnipseed, and J. E. Roybal, "FDA laboratory information Bulletin," LIB 4363, 2005, Laboratory Information Bulletin U.S. Food and Drug Administration, November 2005.

[24] C. Ruan, W. Wang, and B. Gu, "Single-molecule detection of thionine on aggregated gold nanoparticles by surface enhanced Raman scattering," Journal of Raman Spectroscopy, vol. 38, no. 5, pp. 568-573, 2007.

[25] S. He, J. Yao, P. Jiang et al., "Formation of silver nanoparticles and self-assembled two-dimensional ordered superlattice," Langmuir, vol. 17, no. 5, pp. 1571-1575, 2001.

[26] S. Link and M. A. El-Sayed, "Size and temperature dependence of the plasmon absorption of colloidal gold nanoparticles," The Journal of Physical Chemistry B, vol. 103, no. 21, pp. 4212-4217, 1999.

[27] S. D. Solomon, M. Bahadory, A. V. Jeyarajasingam, S. A. Rutkowsky, C. Boritz, and L. Mulfinger, "Synthesis and study of silver nanoparticles," Journal of Chemical Education, vol. 84, no. 2, pp. 322-325, 2007.

[28] C. S. Seney, B. M. Gutzman, and R. H. Goddard, "Correlation of size and surface-enhanced raman scattering activity of optical and spectroscopic properties for silver nanoparticles," The Journal of Physical Chemistry C, vol. 113, no. 1, pp. 74-80, 2009.

[29] Y. Yang, J. Shi, G. Kawamura, and M. Nogami, "Preparation of $\mathrm{Au}-\mathrm{Ag}, \mathrm{Ag}-\mathrm{Au}$ core-shell bimetallic nanoparticles for surfaceenhanced Raman scattering," Scripta Materialia, vol. 58, no. 10, pp. 862-865, 2008.

[30] E. J. Liang, X. L. Ye, and W. Kiefer, "Surface-enhanced Raman spectroscopy of crystal violet in the presence of halide and halate ions with near-infrared wavelength excitation," The 
Journal of Physical Chemistry A, vol. 101, no. 40, pp. 7330-7335, 1997.

[31] K. R. Strehle, D. Cialla, P. Rösch, T. Henkel, M. Köhler, and J. Popp, "A reproducible surface-enhanced Raman spectroscopy approach. Online SERS measurements in a segmented microfluidic system," Analytical Chemistry, vol. 79, no. 4, pp. 15421547, 2007.

[32] Z. Yi, X. Xu, X. Li et al., "Facile preparation of Au/Ag bimetallic hollow nanospheres and its application in surface-enhanced Raman scattering," Applied Surface Science, vol. 258, no. 1, pp. 212-217, 2011.

[33] F.-K. Liu, M.-H. Tsai, Y.-C. Hsu, and T.-C. Chu, "Analytical separation of $\mathrm{Au} / \mathrm{Ag}$ core/shell nanoparticles by capillary electrophoresis," Journal of Chromatography A, vol. 1133, no. 1-2, pp. 340-346, 2006.

[34] C. Langlois, D. Alloyeau, Y. Le Bouar et al., "Growth and structural properties of $\mathrm{CuAg}$ and $\mathrm{CoPt}$ bimetallic nanoparticles," Faraday Discussions, vol. 138, pp. 375-391, 2008.

[35] B. Liu, G. Han, Z. Zhang et al., "Shell thickness-dependent Raman enhancement for rapid identification and detection of pesticide residues at fruit peels," Analytical Chemistry, vol. 84, no. 1, pp. 255-261, 2012.

[36] R. Juluri, A. Rath, A. Ghosh et al., "Coherently embedded Ag nanostructures in Si: 3D imaging and their application to SERS," Scientific Reports, 2014.

[37] L. He, N.-J. Kim, H. Li, Z. Hu, and M. Lin, "Use of a fractal-like gold nanostructure in surface-enhanced Raman spectroscopy for detection of selected food contaminants," Journal of Agricultural and Food Chemistry, vol. 56, no. 21, pp. 9843-9847, 2008.

[38] W. C. Andersen, S. B. Turnipseed, and J. E. Roybal, "Quantitative and confirmatory analyses of malachite green and leucomalachite green residues in fish and shrimp," Journal of Agricultural and Food Chemistry, vol. 54, no. 13, pp. 4517-4523, 2006. 

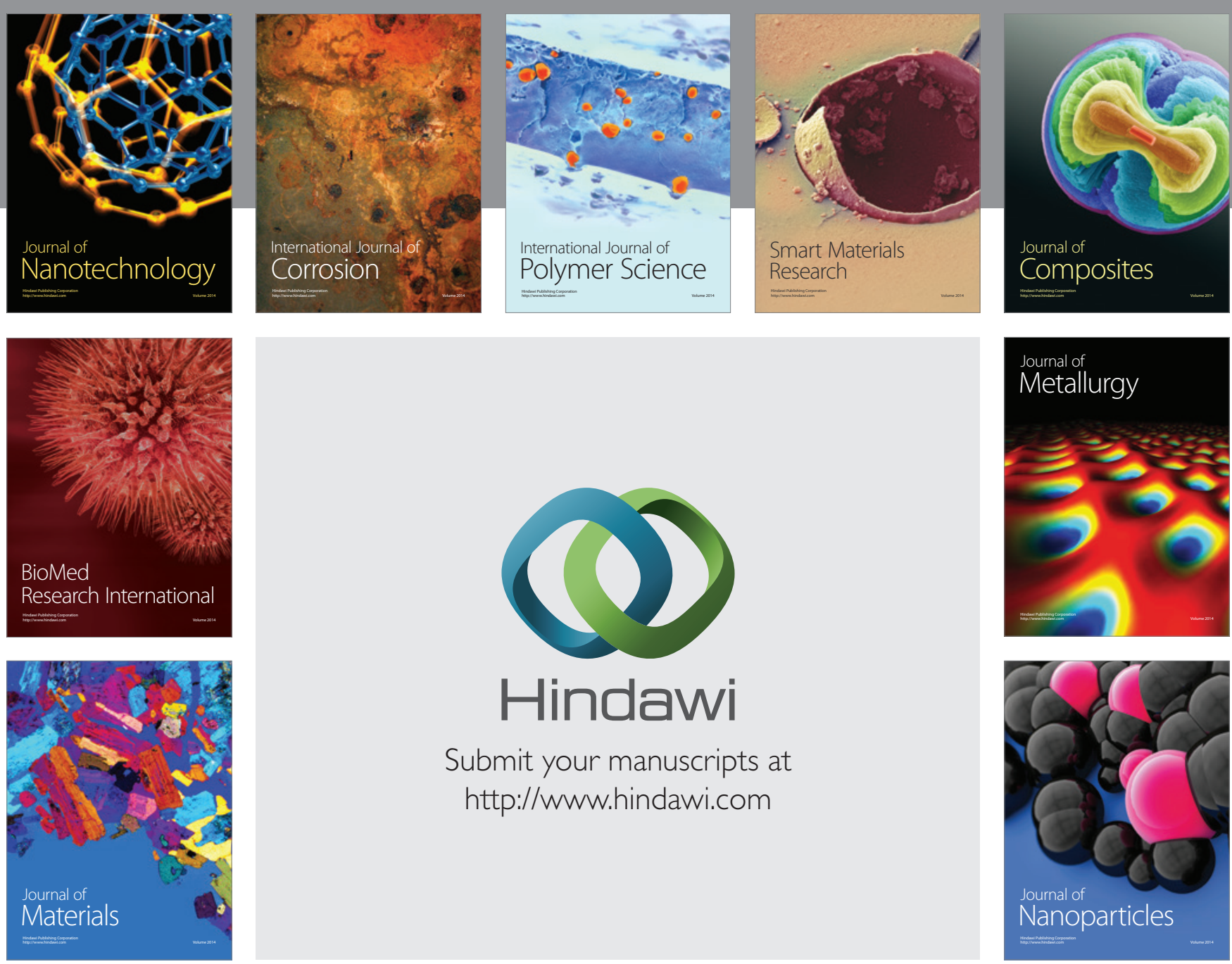

Submit your manuscripts at http://www.hindawi.com
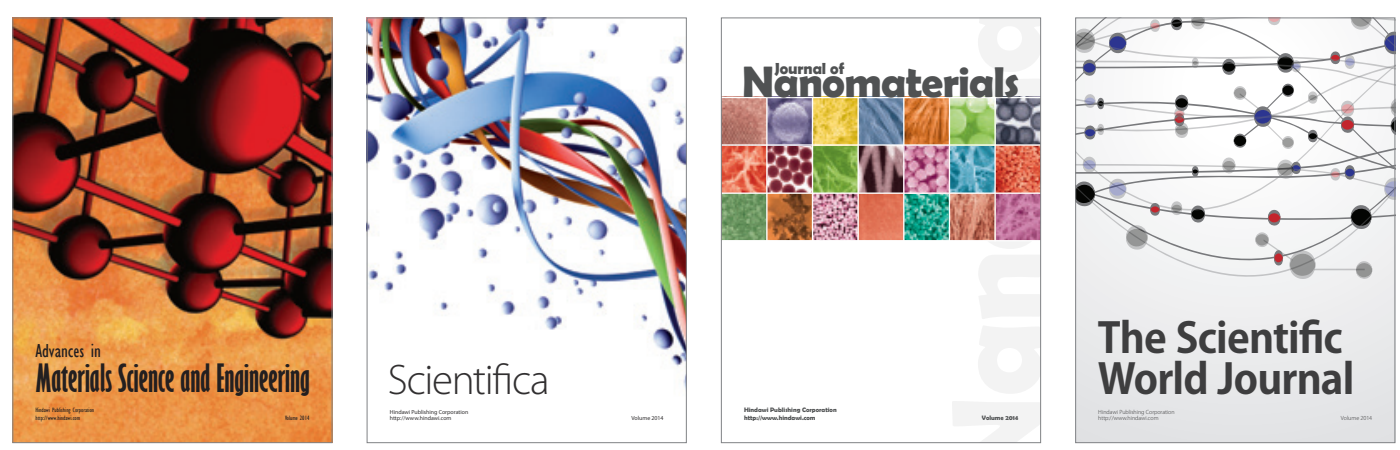

\section{The Scientific World Journal}
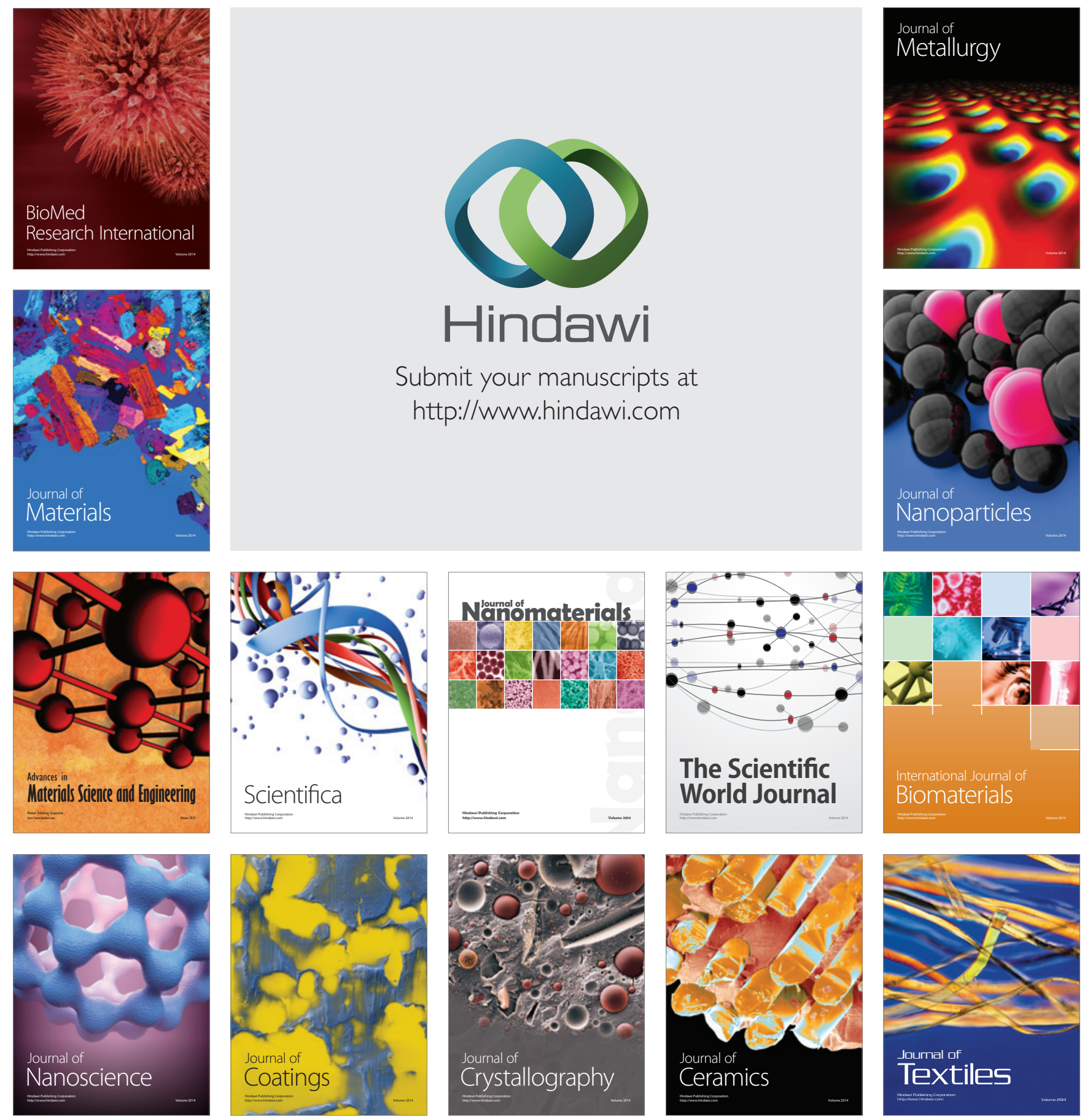